QUARTERLY OF APPLIED MATHEMATICS

VOLUME LXIV, NUMBER 4

DECEMBER 2006, PAGES 735-747

S $0033-569 X(06) 01033-1$

Article electronically published on November 8, 2006

\title{
ELLIPTIC EQUATIONS WITH DIFFUSION COEFFICIENT VANISHING AT THE BOUNDARY: THEORETICAL AND COMPUTATIONAL ASPECTS
}

\author{
BY \\ CHUNG-MIN LEE (Department of Mathematics, Indiana University, Bloomington, Indiana 47405) \\ AND \\ JACOB RUBINSTEIN (Department of Mathematics, Indiana University, Bloomington, Indiana \\ 47405)
}

\begin{abstract}
A class of degenerate elliptic PDEs is considered. Specifically, it is assumed that the diffusion coefficient vanishes on the boundary of the domain. It is shown that if the diffusion coefficient vanishes fast enough, then the problem has a unique solution in the class of smooth functions even if no boundary conditions are supplied. A numerical method is derived to compute solutions for such degenerate equations. The problem is motivated by a certain approach to the recovery of the phase of a wave from intensity measurements.
\end{abstract}

1. Introduction and motivation. Our goal in this paper is to study elliptic partial differential equations, where the diffusion coefficient vanishes at the boundary. We shall present a number of theoretical results on the well-posedness of such problems and a numerical algorithm for solving them.

One motivation to study such problems comes from a basic question in optics: Can the phase of a wave be reconstructed from its intensity? This is an important problem that appears in many applications. In general, measuring the phase is a difficult and expensive process. In contrast to direct phase measurement, measuring the wave's intensity is relatively easy. It is therefore tempting to seek methods for finding the phase from intensity measurements. Indeed, Teague [8] proposed such a phase sensor. His method was further developed by Roddier and others.

To explain the idea behind intensity-based sensors (sometimes called "curvature sensors"), consider a complex-valued wave function $W$ in the Fresnel (paraxial) regime,

Received March 13, 2006.

2000 Mathematics Subject Classification. Primary 35J70.

E-mail address: chunglee@indiana.edu

E-mail address: jrubinst@indiana.edu

(C)2006 Brown University

Reverts to public domain 28 years from publication 
where the wave equation in a homogeneous medium is approximated by

$$
-i \frac{\partial W}{\partial z}=k W+\frac{1}{2 k} \Delta W
$$

Here $z$ is the main direction of propagation, $k$ is the wave number and $\nabla$ and $\Delta$ denote, respectively, the gradient and Laplacian operators in the plane orthogonal to $z$. We use $x$ to denote a point in the plane orthogonal to $z$ (sometimes later on $x$ will be a point in an interval, but no confusion is expected since this will be clear from the context). Writing $W=A e^{i k(z+u)}$ with $A(x, z)$ being the amplitude and $u(x)$ being the phase, we obtain from the imaginary part of (1.1),

$$
-\frac{\partial A}{\partial z}=\frac{1}{2 A} \nabla\left(A^{2} \nabla u\right)
$$

This equation can be written more conveniently as a transport equation for the intensity $I=A^{2}$ :

$$
-\frac{\partial I}{\partial z}=\nabla \cdot(I \nabla u)
$$

The idea behind most intensity-based phase sensors (but not all of them; a different kind of intensity-based phase sensor was proposed in [6]) is to reverse the standard point of view, and to consider (1.3) not as a transport equation for $I$, but rather as an equation for the phase $u$. Teague 8 argued that since (1.3) is, under this interpretation, an elliptic partial differential equation, one should supply suitable boundary conditions for it. In particular, a domain $D$ in which the equation is considered should be specified. He proposed to supplement (1.3) with Dirichlet boundary conditions, in which the values of $u$ on the domain's boundary are given. Roddier [5] addressed the difficulty of obtaining the required boundary values of $u$. He pointed out that in practice the nearly collimated wave passes through an aperture. He therefore suggested considering (1.3) in a domain $D$ that is the image of the aperture on the relevant plane of observation. Then, on the boundary $\partial D$ the intensity will approach zero. He argued (without a mathematical proof) that this implies that $u$ satisfies a homogeneous Neumann boundary condition, i.e.,

$$
\partial_{n} u=0, \quad x \in \partial D,
$$

where $\partial_{n}$ denotes differentiation in the direction of the normal to the boundary. However, there seems to be no mathematical or physical support to this claim.

The fact that $I(x)$ tends to zero as the point of observation $x$ approaches the boundary $\partial D$ makes the elliptic problem (1.3) singular. Our work is inspired by an innovative idea of Gureyev and Nugent [1. They approached the difficulty of missing boundary conditions by using the singular nature of the problem. They assumed that $I$ is a function that is positive in the interior of $D$, while it tends to zero as $x$ approaches the boundary $\partial D$. They claimed that because $I$ vanishes on $\partial D$, no boundary conditions are required for equation (1.3). Their argument went as follows: consider the homogeneous equation

$$
\nabla \cdot(I \nabla v)=0
$$

in $D$. Multiplying the equation by $v$ and integrating by parts gives

$$
\int_{D} I|\nabla v|^{2}-\int_{\partial D} I v \partial_{n} v=0
$$


Since $I=0$ on $\partial D$, they argued, it follows that the second term on the left-hand side of the last equality vanishes, implying that $v$ is constant everywhere in $D$. Thus equation (1.3) has a unique solution (up to an irrelevant additive constant) even without specifying any boundary data on $\partial D$.

However, the uniqueness proof of [1] is not complete. One difficulty is that the integral of $I|\nabla v|^{2}$ may diverge. Yet another difficulty lies in the argument that the boundary term $\int_{\partial D} I v \partial_{n} v$ vanishes because $I$ vanishes on the boundary. The trouble is that although $I$ vanishes on $\partial D$, it does not follow necessarily that this integral vanishes too, since $\partial_{n} v$ might diverge at the boundary.

To demonstrate this point, let us consider the following simple one-dimensional model problem for $v(x)$ where $\gamma$ is a positive parameter:

$$
\left(x^{\gamma} v^{\prime}\right)^{\prime}=0, \quad v(1)=0, \quad 0<x<1 .
$$

Here the singularity is at $x=0$. It is easy to check that this homogeneous problem has a one-parameter family of solutions (we assume for simplicity $\gamma \neq 1$ ):

$$
v(x)=C\left(x^{1-\gamma}-1\right) .
$$

Therefore the problem does not have a unique solution (up to an additive constant). One can now see the difficulty with the argument of [1]. Substituting the solution above into the boundary term $I v \partial_{n} v(x=0)$ shows that this term does not vanish. Notice that for $\gamma<1$ the solutions are bounded, and for $\gamma<1 / 2$ the derivative of the solutions is square integrable.

We conclude that the mathematical nature of equation (1.3) should be more carefully analyzed. In particular, the uniqueness of the solutions and their smoothness depend on the way in which $I$ approaches zero on the boundary. Therefore, we derive in the next section a uniqueness result that distinguishes between different vanishing rates of $I$ at the boundary. Indeed, no boundary condition is needed to determine the unique bounded solution of (1.3) when $I$ vanishes sufficiently fast at the boundary. This raises the question of how to find such a solution. We develop a hybrid analytical-numerical method for this purpose in section 3. In section 4 we report on simulations and tests of the algorithm derived in section 3 . Finally, the results are summarized in section 5 ,

2. A uniqueness theorem. The uniqueness question can be addressed by considering whether the only solutions to the homogeneous problem (1.5) are constants. We assume that the solution $v$ is a bounded function with finite weighted energy, i.e.,

$$
\int_{D} I|\nabla v|^{2}<\infty
$$

We further assume that $D$ is a planar domain with a smooth boundary, and that $I$ is a positive smooth function in $D$, vanishing on the boundary $\partial D$. We prove

Lemma 2.1. Assume there exists a sequence of functions $\xi_{n}(x)$ such that

i). $\xi_{n}=1$ on $\partial D$;

ii). $\xi_{n} \rightarrow 0$ a.e. in $D$;

iii). $\int_{D} I\left|\nabla \xi_{n}\right|^{2} \rightarrow 0$. 
Then equation (1.5) has a unique solution (up to an additive constant) in the class of functions $v$ that are bounded and have finite weighted energy.

Proof. Let $v$ be a solution to (1.5). Standard arguments imply that $v$ minimizes $\int_{D} I|\nabla w|^{2}$ among all functions $w$ such that $w=v$ on $\partial D$.

Let $\xi_{n}$ be a sequence of functions satisfying (i)-(iii) above. We then consider the sequence $u_{n}=\xi_{n} v$. Since on the boundary $\partial D$ we have $u_{n}=v$, it follows that

$$
\int_{D} I\left|\nabla u_{n}\right|^{2} \geq \int_{D} I|\nabla v|^{2}
$$

But

$$
\int_{D} I\left|\nabla u_{n}\right|^{2}=\int_{D} I\left|\nabla\left(\xi_{n} v\right)\right|^{2}=\int_{D} I \xi_{n}^{2}|\nabla v|^{2}+2 \int_{D} I v \xi_{n} \nabla v \cdot \nabla \xi_{n}+\int_{D} I v^{2}\left|\nabla \xi_{n}\right|^{2}
$$

The third term on the right-hand side of (2.3) converges to zero by virtue of property (iii). Using property (iii) once again, together with our assumption (2.1) and the CauchySchwarz inequality we also conclude that the second term converges to zero. Finally, recalling property (ii) we obtain $\int_{D} I \xi_{n}^{2}|\nabla v|^{2} \rightarrow 0$. But (2.2) now implies $\int_{D} I|\nabla v|^{2}=0$, and thus $v$ is constant.

REMARK. The analysis of equation (1.5) by barrier functions (similar to, but not identical to $\xi_{n}$ above) goes back to Khas'minskii, who studied the problem from a probabilistic point of view 3 .

Lemma 2.1 provides a sufficient condition for the uniqueness of solutions to equation (1.3). The condition involves the existence of a sequence $\xi_{n}$ satisfying certain properties. It would be preferable to formulate a condition that is expressed directly in terms of $I$. We therefore formulate and prove a theorem that relates the existence of a sequence $\xi_{n}$ as required in Lemma 2.1 to $I$ itself.

TheOREm 2.2. Assume that $I(x)$ approaches its boundary value uniformly such that $I(x) \sim z^{\gamma}(x)$, where $z(x)=\operatorname{dist}(x, \partial D)$. Then there exists a sequence $\xi_{n}$ satisfying conditions (i)-(iii) of Lemma 2.1 if and only if $\gamma \geq 1$.

Proof. If $\gamma<1$, then $\int_{D} I^{-1}<\infty$. Define for a small positive $\delta$,

$$
D_{\delta}=\{x \in D, \operatorname{dist}(x, \partial D)<\delta\} .
$$

Let $(s, \eta)$ be a local orthogonal coordinate system in $D_{\delta}$, such that $s$ is an arclength parameterization of $\partial D$, and $\eta$ is a coordinate normal to $\partial D$. Assume in contradiction that there exists a sequence $\xi_{n}$ satisfying conditions (i)-(iii) of Lemma 2.1. Denoting the length of $\partial D$ by $|\partial D|$, we can then write for $\delta$ small and $n$ sufficiently large

$$
\begin{aligned}
\frac{|\partial D|}{2} & <\left|\int_{\partial D} \int_{0}^{\delta} \frac{\partial}{\partial \eta} \xi_{n} d \eta d s\right| \leq \int_{\partial D} \int_{0}^{\delta} I^{-1 / 2} I^{1 / 2}\left|\nabla \xi_{n}\right| d \eta d s \\
& \leq\left(\int_{D} I^{-1} \int_{D} I\left|\nabla \xi_{n}\right|^{2}\right)^{1 / 2} .
\end{aligned}
$$

Therefore it cannot be that $\int_{D} I\left|\nabla \xi_{n}\right|^{2} \rightarrow 0$.

Consider now the case $\gamma \geq 1$. Define the sequence of functions $\xi_{n}(x)=1-z^{\alpha_{n}}$, where $z(x)$ was defined above, and $\alpha_{n}$ is any sequence of numbers such that $\alpha_{n} \rightarrow 0$. The 
functions $\xi_{n}$ are smooth, at least for $z$ sufficiently small, and we can truncate them to be 0 at a finite distance $a$ from $\partial D$. Clearly $\xi_{n}(x \in \partial D)=1$, and $\xi_{n} \rightarrow 0$ for $x \in D$. To check property (iii) in Lemma 2.1 we compute

$$
\int_{0}^{a} z^{\gamma}\left(\alpha_{n}^{2} z^{2 \alpha_{n}-2}\right) d z=\alpha_{n}^{2} \int_{0}^{a} z^{2 \alpha_{n}+\gamma-2} d z \rightarrow 0 .
$$

The fact that no sequence $\xi_{n}$ as required in Lemma 2.1 exists when $\gamma<1$ indicates that equation (1.5) does not have by itself a unique solution. This indeed follows from the following theorem [4].

Theorem 2.3. Consider the problem consisting of equation (1.5) together with the boundary condition $v=g$ for $x \in \partial D$ for some boundary data $g$. If $\int_{D} I^{-1}<\infty$, then the problem has a unique solution.

Remark. Notice that Lemma 2.1 and Theorem 2.2 are valid for equations such as (1.5) in any dimension. Theorem 2.3, on the other hand, is proved in [4] only for $D \in R^{2}$. We conjecture that equation (1.5) has no unique solution in any dimension if $\gamma<1$.

3. A hybrid numerical algorithm. Consider the equation

$$
\nabla(I \nabla u)=f, \quad x \in D .
$$

Suppose that $I$ satisfies the conditions of Theorem 2.2 with $\gamma \geq 1$. This means that $\int_{D} I^{-1}$ is unbounded, and thus no boundary condition is needed. How do we solve the singular equation (3.1)? We develop in this section a hybrid analytical-numerical method for this problem. The idea is to integrate (3.1) analytically in a small strip near the boundary $\partial D$, and to derive an effective boundary condition in the reduced domain defined by $D$ minus the strip (see Figure 11).

To perform the local integration we use the $(s, \eta)$ local coordinate system near $\partial D$ that was defined in the previous section. We also denote the tangent and normal vectors at $\partial D$ by $\hat{t}$ and $\hat{n}$, respectively. A strip near $\partial D$ is defined by

$$
Q_{\varepsilon}=\{(s, \eta) \mid 0<\eta<\varepsilon\} .
$$

The boundary $\partial D$ is assumed to be sufficiently smooth so that $Q_{\varepsilon}$ is defined at least for small enough $\varepsilon$. In the strip $Q_{\varepsilon}$ we treat the functions $I, u$ and $f$ as functions of $(s, \eta)$. Denoting the curvature at a boundary point $(s, \eta=0)$ by $\kappa(s)$, the gradient operator can be written as

$$
\nabla u=\frac{1}{1-\kappa \eta} \frac{\partial u}{\partial s} \hat{t}+\frac{\partial u}{\partial \eta} \hat{n}
$$

Similarly we can write the entire PDE (3.1) in local coordinates in $Q_{\varepsilon}$ :

$$
\frac{\partial}{\partial s}\left(I(1-\kappa \eta)^{-1} \frac{\partial u}{\partial s}\right)+\frac{\partial}{\partial \eta}\left((1-\kappa \eta) I \frac{\partial u}{\partial \eta}\right)=(1-\kappa \eta) f .
$$

Integration of equation (3.4) with respect to $\eta$ over the interval $(0, \eta)$ (for some $0<\eta \leq \varepsilon$ ) gives

$$
(1-\kappa \eta) I(s, \eta) \frac{\partial u}{\partial \eta}(s, \eta)=C+\int_{0}^{\eta}(1-\kappa \eta) f d \eta-\int_{0}^{\eta} \frac{\partial}{\partial s}\left(I(1-\kappa \eta)^{-1} \frac{\partial u}{\partial s}\right) d \eta
$$






FIG. 1. The reduced domain

where $C$ is an integration constant. We argue that $C=0$. To see this, divide both sides of (3.5) by $(1-\kappa \eta) I(s, \eta)$, and integrate with respect to $\eta$ over $(0, \varepsilon)$. Since $I^{-1}$ is unbounded, and since $I$ is strictly positive in $D \backslash Q_{\varepsilon}$, it follows that $\int_{Q_{\varepsilon}} I^{-1} d s d \eta$ is unbounded. Therefore, to obtain a bounded solution $u$ we must have $C=0$.

Now substitute $\eta=\varepsilon$ into (3.5), and consider the last term on the right-hand side:

$$
\begin{array}{r}
\int_{0}^{\varepsilon} \frac{\partial}{\partial s}\left(I(s, \eta)(1-\kappa \eta)^{-1} \frac{\partial u}{\partial s}(s, \eta)\right) d \eta=\int_{0}^{\varepsilon} \frac{\partial}{\partial s}\left(I(s, \eta)(1-\kappa \eta)^{-1} \frac{\partial u}{\partial s}(s, \varepsilon)\right) d \eta \\
+O\left(\varepsilon \int_{0}^{\varepsilon} \eta^{\gamma} d \eta\right)
\end{array}
$$

where we assumed that $\frac{\partial u}{\partial s}(s, \eta)$ is a smooth function. Substitution of (3.6) into (3.5), and dividing by $I(s, \varepsilon)(1-\kappa \eta)$ gives (up to $O\left(\varepsilon^{2}\right)$ )

$$
\begin{aligned}
\frac{\partial u}{\partial \eta}(s, \varepsilon)=\frac{1}{I(s, \varepsilon)(1-\kappa \varepsilon)}\left(\int_{0}^{\varepsilon}(1-\kappa \eta) f(s, \eta) d \eta\right. \\
\left.-\int_{0}^{\varepsilon} \frac{\partial}{\partial s}\left(I(s, \eta)(1-\kappa \eta)^{-1} \frac{\partial u}{\partial s}(s, \varepsilon)\right) d \eta\right) .
\end{aligned}
$$

Defining

$$
\Gamma(s, \varepsilon):=\int_{0}^{\varepsilon}\left(I(s, \eta)(1-\kappa \eta)^{-1}\right) d \eta,
$$

and observing that the normal derivative $\partial_{n}$ for the domain $D_{\varepsilon}:=D \backslash Q_{\varepsilon}$ is the negative of the normal derivative on $\partial Q_{\varepsilon}$, we finally arrive at the effective boundary condition for 
$D_{\varepsilon}$ in the form

$$
\frac{\partial u}{\partial n}(s, \varepsilon)=\frac{1}{I(s, \varepsilon)(1-\kappa \varepsilon)}\left(\frac{\partial}{\partial s}\left(\Gamma(s, \varepsilon) \frac{\partial u}{\partial s}(s, \varepsilon)\right)-\int_{0}^{\varepsilon}(1-\kappa \eta) f(s, \eta) d \eta\right) .
$$

To summarize, the hybrid solution method consists of solving the PDE (3.1) in $D_{\varepsilon}$ with the boundary condition (3.9). We devised a finite-elements solver for this problem. Notice that in light of (3.7) there is no gain in using a very fine mesh that gives a numerical error smaller than $O\left(\varepsilon^{2}\right)$. In the next section we report on a number simulation that we carried out.

4. Simulations and numerical tests. 1. A one-dimensional example. We first test the hybrid method in an interval; that is, we solve $\left(x^{\gamma} u^{\prime}\right)^{\prime}=f(x)$ for $x \in(0,1)$ with the boundary condition (at the nonsingular boundary) $u(1)=1$. The discretization norm is $h=1 / N$, where $N$ is the number of nodes used. Although $\varepsilon$ and $h$ are in principle independent of each other (except for the remark in the end of the previous section), we used in this example $\varepsilon=h$. The ordinary differential equation was integrated over the interval $(\varepsilon, 1)$ using the effective boundary condition (3.9). We simulated a number of functions $f(x)$ and obtained similar results for them. For example, we depict in Figure 2 the errors in the weighted norm as a function of the number of nodes for different values of $\gamma$ for the special case $f(x)=1+x+x^{2}$. The exact solution here is

$$
u(x)=1+\frac{1}{2-\gamma}\left(x^{2-\gamma}-1\right)+\frac{1}{2(3-\gamma)}\left(x^{3-\gamma}-1\right)+\frac{1}{3(4-\gamma)}\left(x^{4-\gamma}-1\right),
$$

where $\gamma>1$ is not an integer. The weighted norm is defined as

$$
\|e\|_{w}=\left(\int_{D_{\varepsilon}} I|\nabla e|^{2} d x\right)^{1 / 2} /\left(\int_{D_{\varepsilon}} I d x\right)^{1 / 2}
$$

where $e(x)$ is the difference between the exact $u$ and the computed $u$.

The error is seen to increase with increasing $\gamma$. The reason is that the derivative of $u(x)$ becomes singular when $\gamma>1$.

2. The unit square. We solved (3.1) for the case where $D$ is the unit square $(0,1) \times$ $(0,1)$, and $I(x, y)=x^{\gamma}$. This means that only the part of the boundary along the $y$-axis is singular. The boundary conditions on the other components of the boundary are

$$
u(1, y)=\cos (4) \cos (2 \pi y) \text { for } 0 \leq y \leq 1, \quad u_{y}(x, 1)=u_{y}(x, 0)=0 \text { for } 0 \leq x \leq 1 .
$$

The simulation on which we report here used the function $u(x, y)=\cos (4 x) \cos (2 \pi y)$, but the results are similar for other test functions. The value of $\varepsilon$ was set to be 0.025 in this test.

The dashed and dotted lines in Figure 3 are least-square fitting curves for the error as a function of the number of nodes. The convergence rates that correspond to $\gamma=1.2,1.5,2.0$ and 2.5 are $O\left(N^{-0.4507}\right), O\left(N^{-0.5046}\right), O\left(N^{-0.5215}\right)$ and $O\left(N^{-0.5192}\right)$, respectively, where $N$ represents the number of nodes used in the finite-elements code. We can see that when $\gamma$ increases, that is, when the intensity goes to zero faster, the error decreases. 
3. An optical simulation in a disc. In this test we solved the optical transport equation (1.3) with intensity vanishing on the boundary of the unit disc. The optical simulation was geometrical, though. The hybrid boundary condition (3.6) is used at the artificial boundary. The intensity $I(x, y)$ at a plane $z=z_{0}$ was $\left(1-\sqrt{x^{2}+y^{2}}\right)^{\gamma}\left(\gamma \sqrt{x^{2}+y^{2}}+1\right)$, and the phase $u(x)$ on the same plane is the nonsymmetric function $0.01\left((x / 2)^{2}+y^{2}\right)$. The intensities at $z=z_{0} \pm 1 / 64$ were simulated using the actual phase function, and we then applied a finite difference approximation to find $\partial I / \partial z$. We chose $\varepsilon$ to be 0.025 just as in the previous test. The weighted norm errors for $\gamma=1.2,1.5,2.0$ and 2.5 are shown in Figure 4. The convergence rates for $\gamma=1.2,1.5,2.0,2.5$ with respect to the number of nodes $N$ are $O\left(N^{-0.1928}\right), O\left(N^{-0.6315}\right), O\left(N^{-0.8501}\right), O\left(N^{-1.0963}\right)$, respectively. These results are similar to the previous square test in the sense that when the intensity decreases faster, the reconstructed phase is more accurate. The computed phase and its error in the case of $\gamma=2.5$ with 1206 nodes is shown in Figure 5

4. A very small but nonvanishing diffusion coefficient (at the boundary). The requirement that $I$ vanishes at the boundary is severe for real data. Because of noise and diffraction effects, for example, the intensity is never zero. Theoretically this means that boundary conditions are needed. On the other hand, one might expect the hybrid method to give a reasonable approximation also in such a case. Let us look again at a
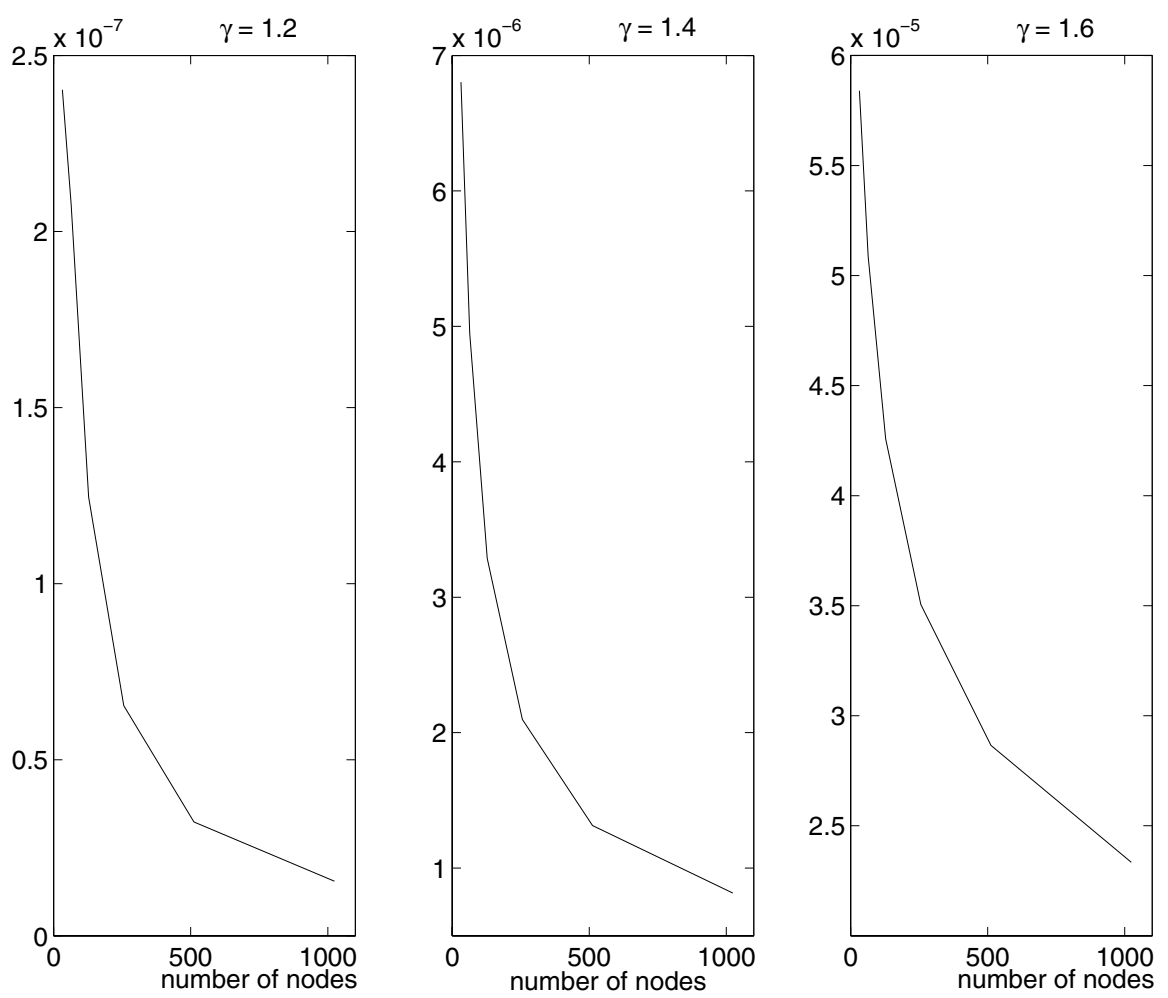

FIG. 2. Errors in the weighted norm in the one-dimensional example for $\gamma=1.2,1.4$ and 1.6 


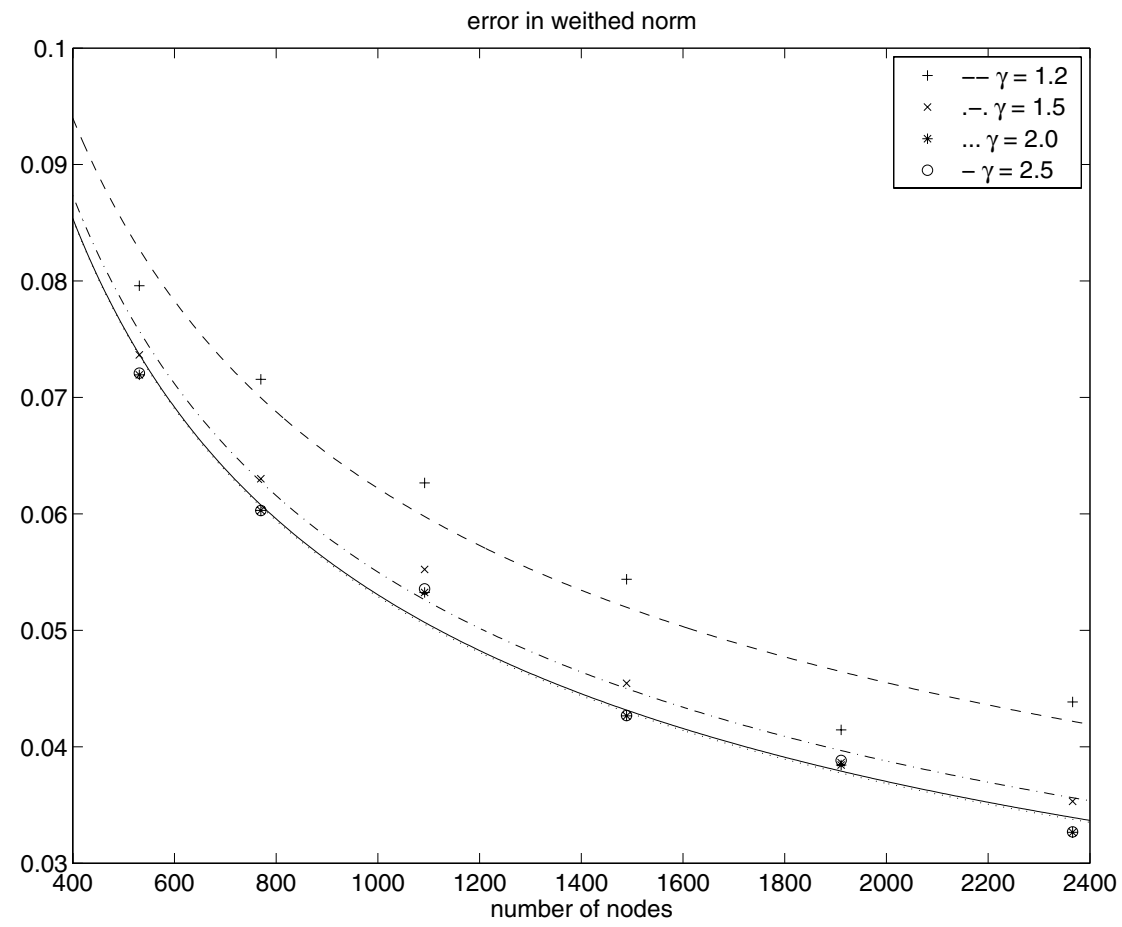

FIG. 3. Errors in the weighted norm for the case where $D$ is the unit square for $\gamma=1.2,1.5,2.0$, and 2.5

one-dimensional example. The underlying model is

$$
\left(I u^{\prime}\right)^{\prime}=f, \quad \gamma>1, \quad x \in(0,1), \quad u(1)=A .
$$

Selecting a boundary layer of thickness $\varepsilon$ near $x=0$ we write, just as before, the effective boundary condition

$$
u^{\prime}(\varepsilon)=\frac{1}{I(\varepsilon)} \int_{0}^{\varepsilon} f(t) d t=\frac{1}{\alpha \varepsilon^{\gamma}} \int_{0}^{\varepsilon} f(t) d t .
$$

Assume now that $I$ is slightly perturbed at $x=0$ by a small positive parameter $\delta$. Thus, (4.1) is replaced in the boundary layer near $x=0$ by

$$
\left(\left(\delta+\alpha x^{\gamma}\right) u^{\prime}\right)^{\prime}=f, \quad x \in(0, \varepsilon) .
$$

Integrating this equation over the boundary layer gives

$$
u^{\prime}(\varepsilon)=\frac{\delta u^{\prime}(0)}{\delta+\alpha \varepsilon^{\gamma}}+\frac{1}{\delta+\alpha \varepsilon^{\gamma}} \int_{0}^{\varepsilon} f(t) d t
$$

One option (method I) is to use the effective boundary condition (4.2), i.e., a boundary condition in which the first term on the right-hand side of (4.4) is neglected. A second option (method II) is to subtract the excess boundary intensity $\delta$ from $I$ everywhere. This brings us back to the case where $I$ vanishes at the boundary. The danger with 




FIG. 4. Errors in the weighted norm when $\gamma=1.2,1.5,2.0$, and 2.5

method I is that the first term on the right-hand side of (4.4) might dominate the second term there. We therefore opt to use method II.

We simulated an optical situation with a radially symmetric wave. The wave at the aperture at $z=0$ is $e^{-4 r^{2}} e^{i k 0.01 \cos (r)}$. The wave number is $k=200$. The intensities at $z=50-1 / 64,50,50+1 / 64$ and the phase at $z=50$ were computed with the RayleighSommerfeld formula. The domain is a disc with radius 2 . We used $\varepsilon=0.025$. In Table 1 we compare the hybrid method as applied to the original data (method I above) to the results obtained by using method II.

TABLE 1

\begin{tabular}{||c|ccccc||}
\hline Nodes & 915 & 1435 & 1992 & 2913 & 3566 \\
\hline Method I & $7.78 \times 10^{-4}$ & $7.29 \times 10^{-4}$ & $7.21 \times 10^{-4}$ & $7.12 \times 10^{-4}$ & $7.09 \times 10^{-4}$ \\
\hline Method II & $3.03 \times 10^{-4}$ & $2.01 \times 10^{-4}$ & $1.80 \times 10^{-4}$ & $1.39 \times 10^{-4}$ & $1.39 \times 10^{-4}$ \\
\hline
\end{tabular}

The convergence rates of the weighted error norm are $O\left(N^{-0.0636}\right)$ using the original intensity (method I) and $O\left(N^{-0.5747}\right.$ ) after subtracting $\delta$ from the intensity everywhere (method II). We see that the "subtraction" method works well in this example. To give an idea of the parameters involved in this example, we provide the values of $\delta=$ $1.1666 \times 10^{-4}, \delta+\alpha \varepsilon^{\gamma}=1.4351 \times 10^{-4}, u^{\prime}(2)=3.888 \times 10^{-2}$, and $u^{\prime}(2-\varepsilon)=3.785 \times 10^{-2}$. 

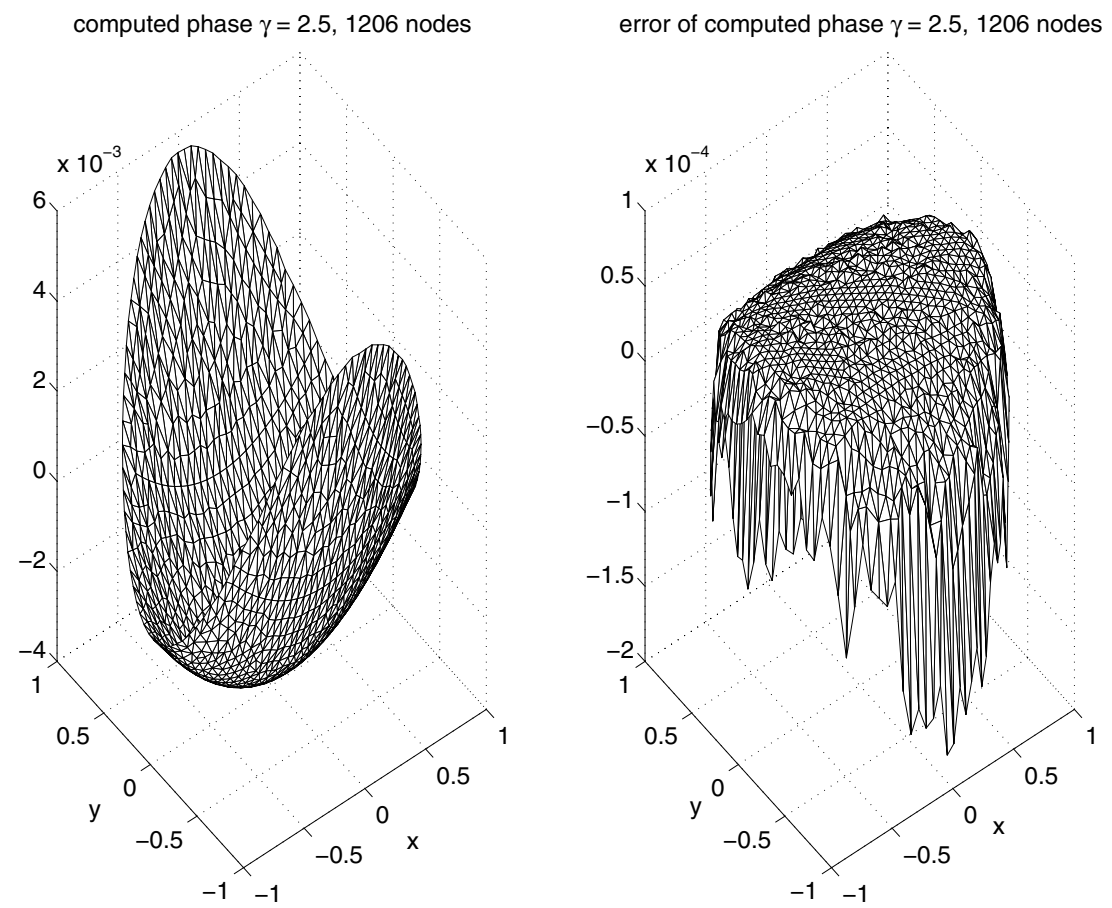

FIG. 5. The computed phase and its error on the unit disc

5. Comparison of the hybrid method to a spectral method. An alternative numerical approach was proposed in reference [1. Gureyev and Nugent used there a spectral expansion method. We compared the hybrid method and the spectral method for a few domains and a few test functions. We present here a one-dimensional example. The following model is used:

$$
\left(I u^{\prime}\right)^{\prime}=f, \quad x \in(0,1), \quad I(x)=x^{2}(1-x)^{2} .
$$

In the current test the exact solution is $u(x)=-\sin (4 x)$, which should not be hard for a spectral method to discern. The convergence of the two methods is compared in Figure 6. We see that the hybrid method gives faster convergence. Similar comparisons hold for other examples that we tested in one-dimensional and two-dimensional domains and in different settings.

5. Summary. We presented a theoretical analysis for the problem of elliptic PDEs with degenerate diffusion coefficient. In addition, we provided a hybrid analyticalnumerical method for computing solutions to such equations. Our main motivation was the problem of phase reconstruction from intensity measurements that appears in a number of optical applications. Therefore, we used this problem as a guide in both the theoretical and numerical part. We report on a number of experiments in which we tested the hybrid method in several setups. We also compared it to a spectral method that was suggested earlier for such problems. 

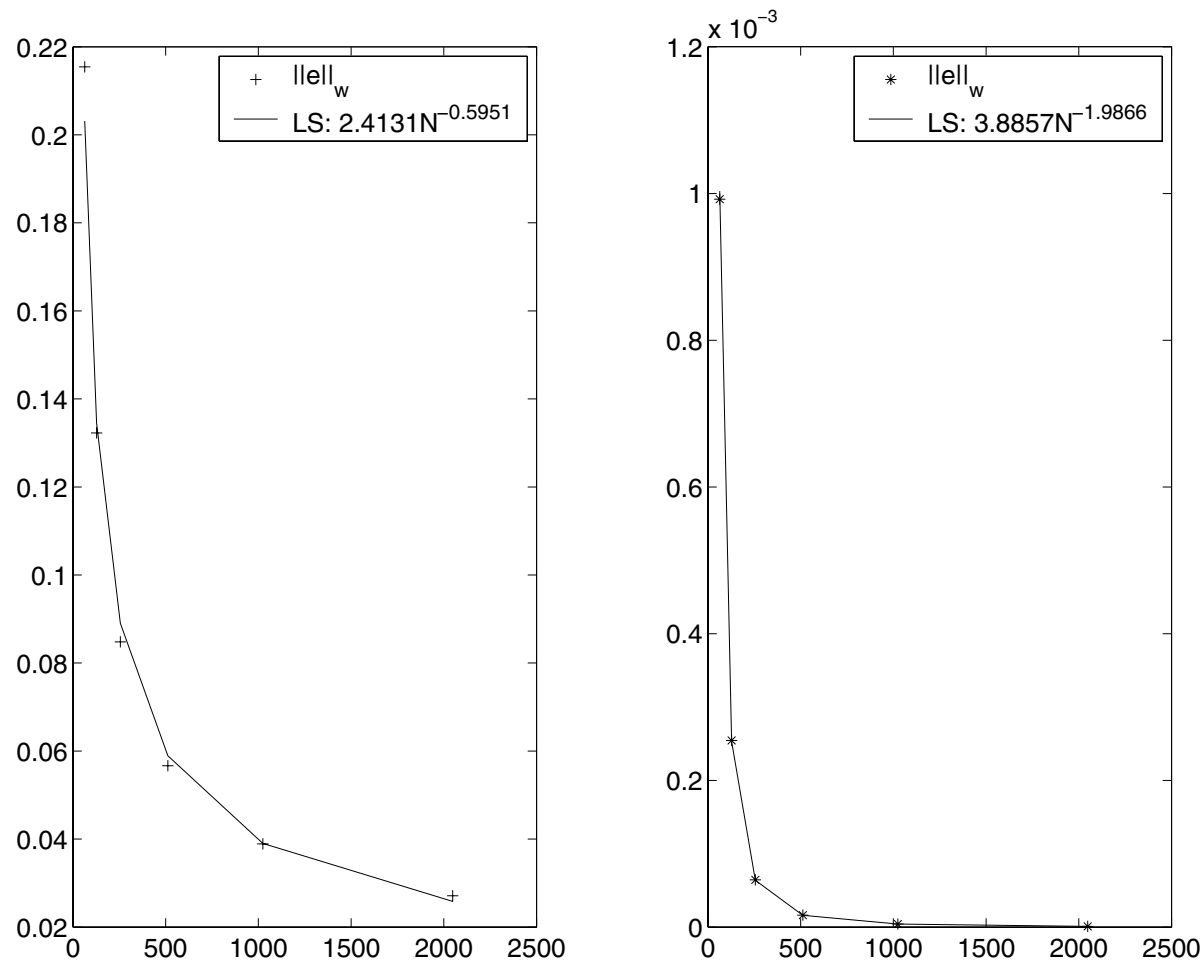

FIG. 6. The convergence of the spectral method (left) and the hybrid method (right) for a one-dimensional example

In addition to our optical motivation, problems where the diffusion coefficient tends to zero at least at part of the boundary arise in other applications. For example, Shiga [7] and Houseworth et al. 2] considered such situations arising in continuum models in genetics.

Acknowledgments. We thank S. Serfati for a useful discussion of this problem. We also thank M. Friedlin for bringing reference 3 to our attention.

\section{REFERENCES}

[1] T.E. Gureyev and K.A. Nugent, Phase retrieval with the transport of intensity equation II: Orthogonal series solution for nonuniform illumination, J. Opt. Soc. Amer. A 13 (1995), 1670-1682.

[2] E. Houseworth, M.S. Jolly and G.O. Mohler, Continuum limit of a discrete genetic problem, preprint.

[3] R. Z. Khas'minskii, Diffusion processes and elliptic differential equations degenerating at the boundary of the domain, Theory of Probability and its Applications 3 (1958), 400-419.

[4] M.K.V. Murthy and G. Stampacchia, Boundary value problems for some degenerate-elliptic operators, Annali di Matematica, LXXX (1968), 1-122. MR0249828(40:3069)

[5] F. Roddier, Curvature sensing and compensation: A new concept in adaptive optics, Appl. Opt. 27 (1998), 1223-1225.

[6] J. Rubinstein and G. Wolansky, A variational principle in optics, J. Opt. Soc. Amer. A 21 (2004), 2164-2172. MR2122591(2005i:35257) 
[7] T. Shiga, Continuous time multi-allelic stepping stone models in population genetics, J. Math. Kyoto Univ. 22 (1982), 1-40. MR0648554 (84h:60138)

[8] M.R. Teague, Deterministic phase retrieval: A Green's function solution, J. Opt. Soc. Amer. 73 (1983), 1434-1441. 